\title{
The entropy increase during the black hole formation
}

\section{Tetsuya Hara, Keita Sakai, Shuhei Kunitomo and Daigo Kajiura}

Department of Physics, Kyoto Sangyo University, Kyoto 603-8555, Japan

email: hara@cc.kyoto-su.ac.japan

\begin{abstract}
The entropy increases enormously when a star collapses into a black hole. For the radiation dominated star, the temperature has decreased from the almost gravitational equilibrium one to the so-called black hole temperature. If we interpret this temperature decrease as the volume increase of the black hole, the entropy increase could be understood as the free expansion of radiation through the increased volume. The expected volume size is tentatively estimated. Under this interpretation, it is derived that the entropy is proportional to the horizon surface area and the microscopic states of the black hole entropy could be understood as the statistical states of the enlarged phase space.
\end{abstract}

Keywords. Uncertainty principle - black hole entropy - entropy increase

\section{Introduction}

Since the research of black hole thermodynamics was born a little over three decades ago several striking concepts have been accepted - see Bekenstein (1973); Hawking (1975); Wald (1998). One of them is that the black hole entropy is proportional to the surface area. It has been insisted that superstring theory could explain the microscopic states of the black hole entropy. However there are still questions about the black hole entropy in its microscopic interpretation. We investigate this problem through the point that the entropy has increased enormously during the black hole formation.

\section{Entropy before black hole formation}

The spherical massive star is considered to collapse due to gravitational instability for its radiation dominance. As the gravitational energy $\left(\Omega=-3 G M / 2 r^{2}\right)$ is almost equal to the thermal energy $\left(U \simeq \epsilon V_{\star}\right)$ for radiation dominated star $(\Omega+U=0)$, let take the relation $3 G M^{2} / 2 r \simeq \epsilon V_{\star}$, where $M, V_{\star}$ and $\epsilon$ are the star mass, volume and radiation energy density, respectively. In the final collapse stage of the black hole formation, the temperature $T\left(=T_{\star}\right)$ is estimated as $T_{\star} \simeq\left(\frac{3 G M^{2}}{2 \tilde{a} r V_{\star}}\right)^{1 / 4} \simeq\left(\frac{3^{3} \cdot 5}{2^{7} \cdot \pi^{3}}\right)^{1 / 4} m_{\mathrm{pl}}\left(\frac{m_{\mathrm{pl}}}{M}\right)^{1 / 2}$ where $r \simeq r_{\mathrm{g}}=2 M, V_{\star}=4 \pi r_{\mathrm{g}}^{3} / 3$, and $m_{\mathrm{pl}}=\sqrt{\hbar c / G}$ are taken and $\epsilon=\tilde{a} T_{\star}^{4}$ is assumed, being $\tilde{a}=\pi^{2} k_{B}^{4} /\left(15 \hbar^{3} c^{3}\right)$. It shows $T_{\star} \propto M^{-1 / 2}$ and the total entropy $S \simeq s V_{\star} \simeq 4 \tilde{a} T_{\star}^{3} V_{\star} / 3 \simeq$ $4 M c^{2} /\left(3 T_{\star}\right)$ is expressed by $S \simeq\left(\frac{2^{3}}{3^{7} \cdot 5}\right)^{1 / 4}\left(\frac{A}{\ell_{\mathrm{pl}}^{2}}\right)^{3 / 4} \simeq 3 \cdot 10^{57}\left(\frac{M}{M_{\odot}}\right)^{3 / 2}$, where $A=4 \pi r_{\mathrm{g}}^{2}$ and $\ell_{\mathrm{pl}}=\sqrt{G \hbar / c^{3}}$ are the black hole surface area and Planck length, respectively. It should be noted that entropy $S$ is proportional to $A^{3 / 4}$, before black hole formation.

\section{Entropy increase and the effective volume of the black hole}

The Bekenstein-Hawking temperature and entropy of a black hole are $T_{\mathrm{BH}}=1 /(8 \pi M)$ and $S=\int d\left(M c^{2}\right) / T_{\mathrm{BH}}=M / 2 T_{\mathrm{BH}}=A /\left(4 l_{p l}^{2}\right) \simeq 10^{77}\left(M / M_{\odot}\right)^{2}$, respectively. Consequently the entropy has increased by an enormous factor $\left(A / \ell_{\mathrm{pl}}^{2}\right)^{1 / 4} \simeq 10^{20}\left(M / M_{\odot}\right)^{1 / 2}$ 
during the black hole formation. Here, let estimate the effective volume $\tilde{V}_{B H}$ of the black hole by assuming $S=s \tilde{V}_{B H}$, where $\tilde{V}_{B H}$ could be interpreted pedagogically to regard Bekenstein-Hawking entropy as an extensive state quantity. Then the volume is inferred as $\tilde{V}_{B H}=\frac{3 A}{4 \cdot 4 \tilde{a} T_{B H}^{3}}=2^{4} \cdot 3^{3} \cdot 5 \cdot \pi \cdot V_{\star}\left(\frac{M}{m_{\mathrm{pl}}}\right)^{2} \propto M^{5}$, where $V_{\star}=4 \pi r_{\mathrm{g}}^{3} / 3$ is used.

The massive star is to collapse even when $r \gg r_{\mathrm{g}}$ and the entropy is almost constant during the contraction before black hole formation, because the contraction of $r \gg r_{\mathrm{g}}$ to $r \simeq r_{\mathrm{g}}$ is almost adiabatic. From the relation $S \simeq M / T$ before and after the black hole formation, this entropy increase is mainly due to the temperature decrease. Before the black hole formation, the temperature is $T_{\star} \propto m_{\mathrm{pl}}\left(m_{\mathrm{pl}} / M\right)^{1 / 2}$ and, after the black hole formation, it becomes $T_{B H} \propto m_{\mathrm{pl}}\left(m_{\mathrm{pl}} / M\right)$.

If the process is free expansion, the temperature changes appropriately as $T \propto(1 / V)^{1 / 4}$, because the internal energy is constant $\left(U \propto T^{4} V=\right.$ const $)$, and/or, thermodynamically, the following relation is satisfied:

$$
\left(\frac{\partial T}{\partial V}\right)_{U}=-\left(\frac{\partial U}{\partial V}\right)_{T} /\left(\frac{\partial U}{\partial T}\right)_{V}=\frac{1}{c_{V} V}\left(p-T\left(\frac{\partial p}{\partial T}\right)_{V}\right)=-\frac{T}{4 V}
$$

where $p$ and $c_{V}$ are pressure and the specific heat at constant volume, respectively. Then the entropy increase could be interpreted as the free expansion of the radiation due to the volume increase by the black hole formation. The expected volume $V_{\mathrm{BH}}$ for the temperature decrease is given by $V_{\mathrm{BH}} \simeq V_{\star}\left(\frac{T_{\star}}{T_{\mathrm{BH}}}\right)^{4} \simeq 2^{3} \cdot 3^{3} \cdot 5 \cdot \pi \cdot V_{\star}\left(\frac{M}{m_{\mathrm{pl}}}\right)^{2} \propto M^{5}$, where $V_{\mathrm{BH}}$ is the effective black hole volume or the effective volume of the gravitationally collapsed object. It is noted that $V_{\mathrm{BH}}$ is almost equal to $\tilde{V}_{\mathrm{BH}}$, so we use $V_{\mathrm{BH}}=\tilde{V}_{\mathrm{BH}}$ to denote the assumed effective volume of the black hole.

The interpretation of the volume increase $V_{\mathrm{BH}} \simeq V_{\star}\left(M / m_{\mathrm{pl}}\right)^{2}$ is supplemented by the following consideration. The uncertainty principle $\Delta x \Delta p \geqslant \hbar$ shows one massless particle energy $\Delta e$ should satisfy the relation $\Delta e \simeq \Delta p c \geqslant \hbar c / \Delta x$. Taking $\Delta x \simeq r_{g}=2 M$, the upper limit of the particle number is given by $N_{\mathrm{BH}} \simeq M /(\Delta e) \leqslant G M^{2} /(\hbar c)=\left(M / m_{\mathrm{pl}}\right)^{2}$. On the other hand, the number of single particle quantum states in phase space of a black hole is given by $n_{\mathrm{BH}} \simeq V_{\mathrm{BH}}(\Delta p)^{3} / \hbar^{3} \simeq\left(M / m_{\mathrm{pl}}\right)^{2}$, where $V_{\mathrm{BH}} \simeq V_{\star}\left(M / m_{\mathrm{pl}}\right)^{2}$ and $V_{\star} \simeq(\Delta x)^{3}$ are used. Then the number of ways $W$ to distribute the $N_{\mathrm{BH}}$ particles among the $n_{\mathrm{BH}}$ states is $W=\left(N_{\mathrm{BH}}+n_{\mathrm{BH}}-1\right) ! /\left(N_{\mathrm{BH}} !\left(n_{\mathrm{BH}}-1\right) !\right)$. Taking Stirling's formula $\ln n ! \simeq n \ln n-n$, the entropy becomes as $S=\ln W \simeq N_{B H}((1+\alpha) \ln (1+\alpha)-\alpha \ln \alpha) \propto$ $N_{B H} \simeq\left(M / m_{\mathrm{pl}}\right)^{2} \simeq A / l_{\mathrm{pl}}^{2}$, where $N_{B H} \simeq \alpha n_{B H}(\gg 1)$ is taken.

\section{Discussion and Conclusions}

If we accept the increase of the effective black hole volume, the microscopic states of black hole entropy could be interpreted as the statistical state number in the phase space and the entropy could be taken as the extensive state quantity. The enormous entropy increase and temperature decrease in the black hole formation could be understandable as the free expansion of composed particles to this increased black hole volume.

\section{References}

Bekenstein, J. D. 1973, Phys. Rev., D7, 2333

Hawking, S. W. 1975, Commun. Math. Phys., 43, 199

Wald, R. M. 1998, Black Holes and Relativistic Stars (Chicago Univ. Press), gr-qc/9912119

Frolov, V. P. \& Novikov, I. D. 1998, Black Hole Physics (Kluwer: Dordrecht)

Mukohyama, S. 1998, PhD Thesis (Kyoto University), gr-qc/9812079

Page, D. N. 2004, hep-th/0409024

Kiefer, C. 2002, astro-ph/0202032 